\title{
Optimal Allocation of COVID-19 Test Kits Among Accredited Testing Centers in the Philippines
}

\author{
Christian Alvin H. Buhat ${ }^{1}$ (D) . Jessa Camille C. Duero ${ }^{1} \cdot$ Edd Francis O. Felix $^{1}$. \\ Jomar F. Rabajante ${ }^{1,2}$. Jonathan B. Mamplata ${ }^{1}$
}

Received: 23 April 2020 / Revised: 3 September 2020 / Accepted: 7 October 2020 /

Published online: 9 November 2020

(C) Springer Nature Switzerland AG 2020

\begin{abstract}
Testing is crucial for early detection, isolation, and treatment of coronavirus disease (COVID-19)-infected individuals. However, in resource-constrained countries such as the Philippines, test kits have limited availability. As of 11 April 2020, there are 11 testing centers in the country that have been accredited by the Department of Health (DOH) to conduct testing. In this paper, we use nonlinear programming (NLP) to determine the optimal percentage allocation of COVID-19 test kits among accredited testing centers in the Philippines that gives an equitable chance to all infected individuals to be tested. Heterogeneity in testing accessibility, population density of municipalities, and the capacity of testing facilities are included in the model. Our results show that the range of optimal allocation per testing center are as follows: Research Institute for Tropical Medicine (4.17-6.34\%), San Lazaro Hospital (14.65$24.03 \%$ ), University of the Philippines-National Institutes of Health (16.25-44.80\%), Lung Center of the Philippines (15.8-26.40\%), Baguio General Hospital Medical Center (0.58-0.76\%), The Medical City, Pasig City (5.96-25.51\%), St. Luke's Medical Center, Quezon City (1.09-6.70\%), Bicol Public Health Laboratory (0.06$0.08 \%)$, Western Visayas Medical Center (0.71-4.52\%), Vicente Sotto Memorial Medical Center (1.02-2.61\%), and Southern Philippines Medical Center $(\approx 0.01 \%)$. Our results can serve as a guide to the authorities in distributing the COVID-19 test kits. These can also be used for proposing additional testing centers and utilizing the available test kits properly and equitably, which helps in "flattening" the epidemic curve.
\end{abstract}

Keywords Coronavirus · Optimal allocation · Test kits · Nonlinear programming · Infectious diseases · COVID-19

Christian Alvin H. Buhat

chbuhat@up.edu.ph

1 Institute of Mathematical Sciences and Physics, University of the Philippines Los Baños, 4031, Laguna, Philippines

2 Faculty of Education, University of the Philippines Open University, 4031, Laguna, Philippines 


\section{Introduction}

The coronavirus disease (COVID-19) is an infectious respiratory disease caused by the novel coronavirus strain officially known as severe acute respiratory syndrome coronavirus 2 (SARS-Cov-2) [25]. The virus is said to have originated from Wuhan, China [32], and affected hundreds of thousands of people across 146 countries as of mid-March [9]. The World Health Organization (WHO) officially declared the COVID-19 as a global pandemic [20].

Countries with widespread testing and surveillance strategies, like South Korea and Germany, have been able to contain the spread the virus [5, 10, 15, 25]. Implementation of extensive testing for COVID-19 is essential to help researchers understand the epidemiology of the disease. It may also allow them to trace even asymptomatic cases that can accelerate the increase in the number of cases and cause epidemic control problems. More importantly, this helps policymakers and healthcare professionals reduce spread, strategically deliver treatment resources, and devise appropriate policy responses [16, 17, 21, 27].

In the Philippines, the Department of Health $(\mathrm{DOH})$ is enhancing its coronavirus laboratory testing capacity, hospital preparedness, rapid response, and its risk communication and information dissemination [12]. The government plans to start a targeted massive testing program for COVID-19 starting April 14 to fully assess the public health situation [13]. However, there have been substantial constraints in resources and other challenges with conducting adequate testing, as the testing capacity is overburdened that even the well-resourced nations were overwhelmed by the effects of the pandemic $[14,17,25]$. These challenges with large-scale testing are exacerbated in resource-constrained countries such as the Philippines. Although DOH plans to significantly increase the capacity for testing, resources, such as test kits, remain insufficient to test a large portion of the susceptible population in the country [22]. As of April 11, 2020, the Philippines has reported about 5000 confirmed cases from the 38,640 COVID-19 tests conducted by the $11 \mathrm{DOH}$-accredited laboratories and hospitals in the country as shown in Fig. 1 [19]. This relatively low number of reported cases could be due to difficulty in extending the tests to the population most in need, and a meager supply of testing resources to identify COVID-19 cases $[7,30]$. Thus, it is necessary for the Philippines to come up with an optimal strategy for distributing available test kits among the testing centers so that each individual suspected to be infected has an equal chance of being tested.

In this paper, we use a nonlinear programming (NLP) model on designing equitable resource allocation on resource-constrained countries to address this issue. The mathematical model includes heterogeneity in testing accessibility and the data are population density-based to incorporate the different conditions on each communities where people in dense communities have more face-to-face interaction and thus are most at risk in getting infected $[11,29]$. The equity objective function of the model provides an equal assessment of the distribution of the limited test kits among the testing centers. We apply the model to the population density-adjusted number of COVID-19-infected individual projection in the Philippines. We let the basic reproduction number (average number of direct secondary cases generated from a 


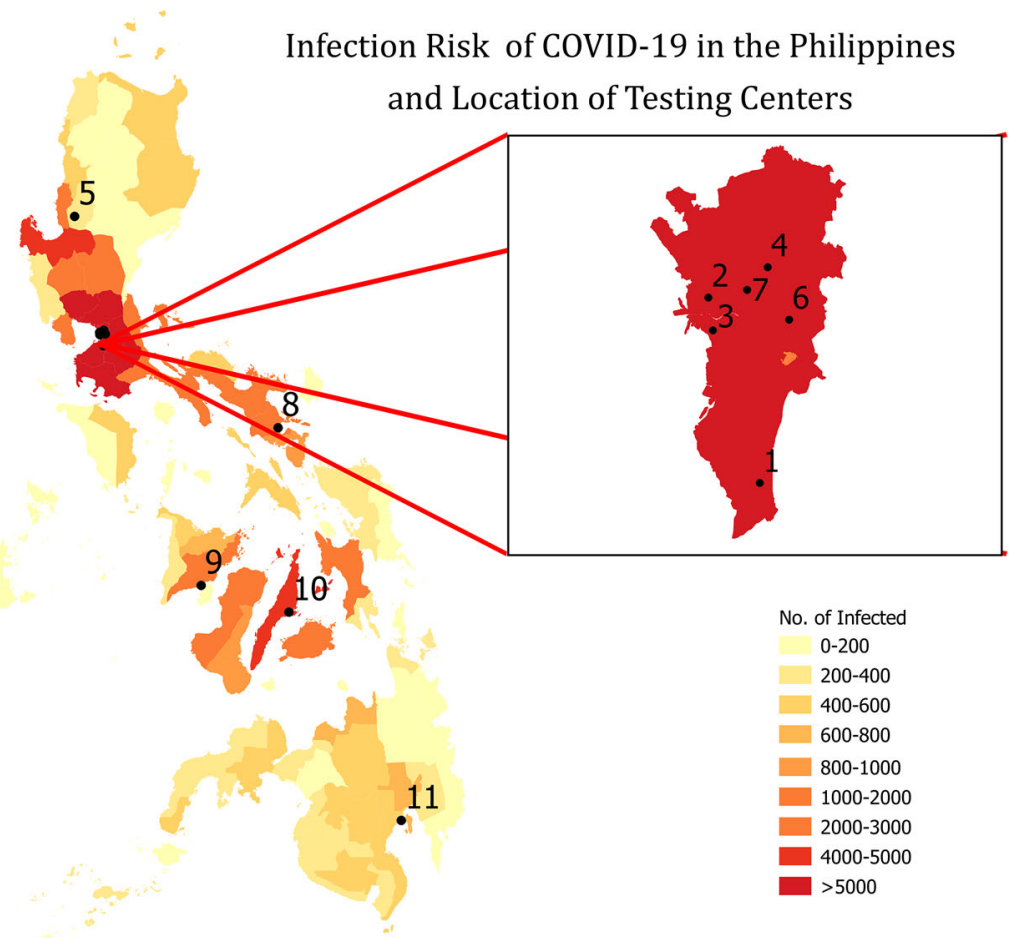

Fig. 1 Heat map of infections and locations of testing centers accredited by the World Health Organization as of 11 April 2020. The following are the accredited testing centers and are shown on the map: (1) Research Institute for Tropical Medicine (RITM); (2) San Lazaro Hospital; (3) University of the Philippines - National Institutes of Health (UP-NIH); (4) Lung Center of the Philippines; (5) Baguio General Hospital and Medical Center (BGHMC); (6) The Medical City, Pasig City (TMC); (7) St. Luke's Medical Center, Quezon City; (8) Bicol Public Health Laboratory (BPHL); (9) Western Visayas Medical Center (WVMC); (10) Vicente Sotto Memorial Medical Center (VSMMC); (11) Southern Philippines Medical Center (SPMC

contagious person in a fully susceptible community) of COVID-19 in the country to be $R_{0}=2.5$ to estimate the infected number of individuals that should be tested [4, 23]. We consider the population of each municipalities in the country as well as the current testing centers accredited by the DOH.

The remaining parts of the paper are organized as follows: in Section 2, we discuss the preliminaries of the model: estimating the number of infected individuals, accessibility of test kits, and a model for the distribution of test kits. We also develop the constraints which is then utilized in the NLP model formulated. In Section 3, we find the coordinates for the communities and determine the different values for the dispersal length scale parameter $k$. These values are used to determine the optimal allocation of test kit distribution. Section 4 interprets the results and implications of the study. Section 5 presents conclusion and offers recommendations. The last section provides the supplementary materials of the paper. 


\section{Model Formulation}

\subsection{Preliminaries}

In this model, we consider the municipalities and cities in the Philippines to be the community $i$ and estimate the number of infected individual in each community. We assume that these individuals will go to the nearest accredited testing center $j$ in terms of great circle distance and only one test kit will be used per individual.

\subsubsection{Estimating the Number of Infected Individuals}

We consider a forecasted population of each communities in the Philippines for the year 2019 based on the 2015 Philippine Statistics Authority (PSA) Census [1]. Each population of the community is then multiplied to $1-\frac{1}{R_{0}}$ with $R_{0}=2.5$ where $R_{0}$ is the reproduction number of the virus or the epidemiological metric that describes the contagiousness or transmissibility of an infectious disease [6]. This will give us the approximate cumulative estimate of infected individuals during the whole epidemic period per community on a do-nothing situation in a well-mixed system [24]. To relax the well-mixing property of the model, we then consider the population density of each community, and factor in a 1-m additional distance around a person (or $4 \mathrm{~m}^{2}$ safe space per person) as recommended by the WHO to observe social distancing. Factoring in the density of each communities also allows our result to be applicable for a long period of time due to the stable trend and distribution of population densities and its correlation to the number of infected individuals [11]. Thus, we compute for the projected total number of infected individuals in community $i, I_{i}$, as:

$$
\begin{aligned}
I_{i}= & \text { Population size in community } i \times\left(1-\frac{1}{R_{0}}\right) \\
& \times \text { Population density for community } i \times \frac{4 m^{2}}{1 \text { person }},
\end{aligned}
$$

where population density is the number of people per one million square meter.

\subsubsection{Computing for the Testing Accessibility}

We assume that an individual tends to get tested in the nearest testing center $j$. The lesser the distance between the testing center and the person's community, the greater the chance of the person going to that testing center. Due to data insufficiency in road and sea networks in the country's archipelago, we consider the great circle distance or the shortest distance between two points over the Earth's surface [18]. To provide precise distances, we use a spherical law of cosines formula [28], and let $d_{i j}$ be the distance between community $i$ and testing center $j$ expressed as:

$$
d_{i j}=\operatorname{Arccos}\left(\cos \left(\mathrm{lat}_{i}\right) \cos \left(\mathrm{lat}_{j}\right) \cos \left(\operatorname{lon}_{j}-\operatorname{lon}_{i}\right)+\sin \left(\mathrm{lat}_{i}\right) \sin \left(\mathrm{lat}_{j}\right)\right) \times R,
$$

where $R$ is the Earth radius $(6371 \mathrm{~km}), l^{a} t_{i}$ and $l{ } n_{i}$ are the GPS location of community $i, l a t_{j}$ and $l o n_{j}$ are the GPS location of testing center $j$, and all angles are 
in radian measure. Using $d_{i j}$, we then multiply it to the number of estimated infected individuals per community to compute for the "effective demand," $f\left(d_{i j}\right) I_{i}$ or the number of infected individuals in community $i$ that will go to testing center $j$ for testing. We let $f$ be a function that puts "weight" on the testing accessibility of an individual to a testing center based on the distance $d_{i j}$. To model the testing accessibility, we use a Gaussian model, which is used to describe the distribution of the COVID-19 infections [26]. We have $f(d)=e^{-k d^{2}}$, where $k$ is a dispersal length scale parameter that can provide further information about outbreak dynamics and potential for superspreading events [23]. We use Elliot's estimation [8] in solving for $k$ which considers the mean $\mu$, variance $\sigma^{2}$ of the distribution of the infected population, and the sample size $N$ for number of communities involved:

$$
k=\frac{\mu^{2}-\frac{\sigma^{2}}{N}}{\sigma^{2}-\mu} .
$$

\subsubsection{Distribution of Test Kits}

We follow the approach formulated by Wilson and Blower [31] in determining the test kit distribution to each testing centers. First, we compute how many infected individuals will go to a certain testing center $j$ through the "effective demands" $f\left(d_{i j}\right) I_{i}$ for each testing center $j$. We call this the demand $D_{j}$ where

$$
D_{j}=\sum_{i=1}^{m} f\left(d_{i j}\right) I_{i},
$$

which is the sum of the effective demand of all communities to testing center $j$. We then divide $f\left(d_{i j}\right) I_{i}$ to $D_{j}$ to determine the percentage of test kits allocated by a testing center to each community. This is multiplied to the number of test kits allocated to that testing center to determine the number of individuals in each community that can get tested by testing center $j$. We call this $T_{i}^{j}$ where

$$
T_{i}^{j}=S_{j} \frac{f\left(d_{i j}\right) I_{i}}{D_{j}}
$$

where $S_{j}$ is the supply allocation of COVID-19 test kits on testing center $j$ and $\frac{f\left(d_{i j}\right) I_{i}}{D_{j}}$ is the percent allocation of each testing center $j$ to each community $i$. Thus, given that one test kit is assigned per individual, we get the sum of all the number of tested individuals in each community $i$ tested on each testing centers, which gives us the total number of tested individuals in community $i, T_{i}$, where

$$
T_{i}=\sum_{j=1}^{n} T_{i}^{j} .
$$

Each testing center has a maximum number of tests that they can perform per day, and so we also factor in the daily testing limit $L_{j}$ of each testing center, and compute for 
$M$, which is the the maximum number of testing days before all infected individuals can be tested. Thus, we have

$$
M=\frac{\sum_{i=1}^{m} I_{i}}{\sum_{j=1}^{n} L_{j}} .
$$

\subsection{NLP Model}

Nonlinear programming (NLP) is an optimization technique where the objective function is nonlinear and/or the feasible region is determined by nonlinear constraints [2]. Here, we use the NLP model based on Wilson and Blower [31], with COVID19 data specific to the Philippines, to determine the optimal distribution of test kits among the testing centers. The model is described by:

$$
\begin{aligned}
\min E\left(S_{1}, S_{2}, \ldots, S_{n}\right) & =\sum_{i=1}^{m}\left(\frac{T_{i}}{I_{i}}-\frac{A}{I_{T}}\right)^{2} \\
\text { subject to } & \sum_{j=1}^{n} S_{j}=A \\
S_{j} \leq M L_{j}, & j=1, \ldots, n \\
S_{j} & \geq 0, \quad j=1, \ldots, n \\
T_{i} \leq I_{i}, & i=1, \ldots, m
\end{aligned}
$$

where $S_{j}$ is the allocation of test kits to testing center $j, A$ is the total number of test kits to be allocated, and $I_{T}$ is the total number of infected individuals per community. Here, we base the objective function to the Euclidean norm to provide a standard result in comparison with other norms [3]. We use an equity objective function with Euclidean norm to ensure equal opportunities among all infected individuals in getting tested. We minimize the equity objective function $E$, with optimal allocations to each testing center $\left(S_{j}\right)$ such that (i) all available test kits will be used, (ii) daily test limits per testing center will be considered and will not exceed the maximum number of days for testing, (iii) there will be a non-negative supply of test kits per testing center, and (iv) the number of tested individuals in each community is less than the expected maximum number of infected individuals. Each individual will only be subjected to one test, which will serve as an initial testing. We solve this NLP model through simulations in $R$ and explore scenarios such as additional testing centers to communities with $I_{i} \geq 5000$.

\section{Numerical Illustrations}

We determine the GPS coordinates of all the cities and municipalities in the Philippines, and of the 11 accredited testing centers using Geocode by Awesome Table app in google spreadsheets. This app returns the latitude and longitude coordinates of the 
testing centers' locations and the geographic centers of the cities and municipalities. We use these coordinates in the computations except for two: Datu Hoffer Ampatuan and Shariff Sardona Mustapha, Maguindanao. Geocode app cannot locate Shariff Sardona Mustapha, and it returned incorrect coordinates for Datu Hoffer Ampatuan. We use the coordinates of one barangay from each municipality instead. In addition, we compute for various dispersal length scale parameters $k$ shown in (3) using the mean, $\mu$, and variance, $\sigma^{2}$, of distributions of each density structures in the country: (i) population density-based distribution of infected individuals in the whole Philippines which resulted in $k=0.0849$, (ii) distribution of infected individuals in Metro Manila and this results in $k=0.4136$, and (iii) population density-based distribution of infected individuals in Metro Manila that results in $k=1.5614$, the highest density in the country.

We utilize the 11 accredited testing centers to determine the optimal distribution of test kits. Shown in Table 1 is the proportion of test kits to be distributed among the 11 testing centers using different values for $k$. We can observe that UP-NIH will receive the biggest proportion, ranging from 16.25 to $44.80 \%$, while Southern Philippines Medical Center will receive the smallest proportion which is approximately $0.01 \%$.

From the results, we notice that San Lazaro Hospital, UP-NIH, and Lung Center of the Philippines got the majority of the proportion, across all scenarios. If mass testing will push through, these three hospitals will be overworked and will not be able to deliver timely results on the tests. To address this, we propose to add more testing centers. We identify 10 communities with the highest number of infected individuals and no existing accredited testing center. We will treat these 10 communities as the location of the suggested additional testing centers. We then compute for the optimal distribution of test kits to the 21 testing centers. The proportion of test kits allocated to each of UP-NIH, San Lazaro Hospital, Lung Center of the Philippines, and Makati City is greater than $10 \%$. We can say that this distribution poses concerns similar to that of only having 11 testing centers. To reduce the proportion of test kits allocated

Table 1 Proportion of test kits to be distributed in the different testing centers for $k=1.5614, k=0.4136$, and $k=0.0849$ where "*” are assumed values for daily limit

\begin{tabular}{llllll}
\hline Testing center & $k=1.5614$ & $k=0.4136$ & $k=0.0849$ & Range & Daily limit \\
\hline RITM & $6.34 \%$ & $4.17 \%$ & $6.26 \%$ & {$[4.17 \%, 6.34 \%]$} & 900 \\
San Lazaro & $23.4 \%$ & $24.03 \%$ & $14.65 \%$ & {$[14.65 \%, 24.03 \%]$} & 100 \\
UP-NIH & $44.8 \%$ & $16.25 \%$ & $27.79 \%$ & {$[16.25 \%, 44.80 \%]$} & 80 \\
Lung center & $15.8 \%$ & $20.29 \%$ & $26.4 \%$ & {$[15.80 \%, 26.40 \%]$} & 100 \\
BGHMC & $0.76 \%$ & $0.64 \%$ & $0.58 \%$ & {$[0.58 \%, 0.76 \%]$} & 150 \\
TMC & $5.96 \%$ & $25.51 \%$ & $15.65 \%$ & {$[5.96 \%, 25.51 \%]$} & $100^{*}$ \\
St. Luke's & $1.09 \%$ & $1.9 \%$ & $6.7 \%$ & {$[1.09 \%, 6.70 \%]$} & $100^{*}$ \\
BPHL & $0.06 \%$ & $0.07 \%$ & $0.08 \%$ & {$[0.06 \%, 0.08 \%]$} & $100^{*}$ \\
WVMC & $0.77 \%$ & $4.52 \%$ & $0.71 \%$ & {$[0.71 \%, 4.52 \%]$} & 80 \\
VSMMC & $1.02 \%$ & $2.61 \%$ & $1.17 \%$ & {$[1.02 \%, 2.61 \%]$} & 240 \\
SPMC & $0.01 \%$ & $0.01 \%$ & $0.01 \%$ & {$[0.01 \%, 0.01 \%]$} & 100 \\
\hline
\end{tabular}


to each of these testing centers, we consider all communities with more than 5000 infected individuals. We then identify 19 communities as locations of proposed testing centers in addition to the original 11 and compute for the optimal distribution of test kits to the 30 testing centers.

In addition, we obtain various sets of percentage allocations from different values of $k$ representing different population density structures. Each $k$ value from testing centers located in NCR (most populated region) varies in the percentage allocation per $k$ (RITM, San Lazaro, UP-NIH, Lung Center, TMC, and St. Luke's). One reason is that these testing centers have common infected individuals that are approximately close to multiple centers. This gives these individuals the option to go to their preferred testing center. Meanwhile, testing centers outside NCR remain fairly constant in terms of distribution, regardless of the value of $k$, since the same portion of infected individuals will get tested there.

Shown in Fig. 2 are the pie charts representing the optimal distribution of test kits for different number of testing centers and different values of dispersal length parameter. It is evident that as we increase the number of testing centers from 11 to 30 , there is a great decrease of proportions to be distributed on the currently existing testing centers. Newly introduced testing centers will get a substantial amount of test kits and will serve infected individuals in their respective vicinity.

From the optimal solution computed using 30 testing centers, we can see that the maximum proportion of test kits to be distributed is in Navotas City, which accounts for $8.68 \%$ of the total number of testing centers. This is achieved for $k=0.4136$. Shown in Fig. 3 is the map with corresponding locations of the 30 testing centers.

\section{Discussion}

In this paper, we determined an optimal allocation of COVID-19 test kits among the DOH-accredited testing centers in the Philippines. We utilized the formulated NLP model of Wilson and Blower [31], which gave an equitable opportunity to each susceptible individual to be tested. The model involved COVID-19-specific conditions that considered the infected population, the reproduction number of the disease $\left(R_{0}=2.5\right)$, the distribution of infected individuals, and the testing limits of each testing center. We applied this to the municipality/city data of the Philippines and computed the percentage of distribution on various dispersal length parameter, $k$ : $k=0.0849, k=0.4136$, and $k=1.5614$.

We ran the optimization problem in $R$ to determine the optimal allocation of test kits to be distributed among the accredited DOH testing centers and additional testing centers found in highly infected communities in the country. Based on our results, the optimal range of distribution per testing center are as follows: RITM (4.17-6.34\%), San Lazaro (14.65-24.03\%), UP-NIH (16.25-44.80\%), Lung Center (15.8-26.40\%), BGHMC (0.58-0.76\%), TMC (5.96-25.51\%), St. Luke's, QC (1.09-6.70\%), BPHL (0.06-0.08\%), WVMC (0.71-4.52\%), VSMMC (1.02-2.61\%), and SPMC $(\approx 0.01 \%)$. With this allocation, we utilized $100 \%$ of the limited test kits available to the country and distribute it optimally to all testing centers wherein every distribution is proportional to the number of infected people around that testing 

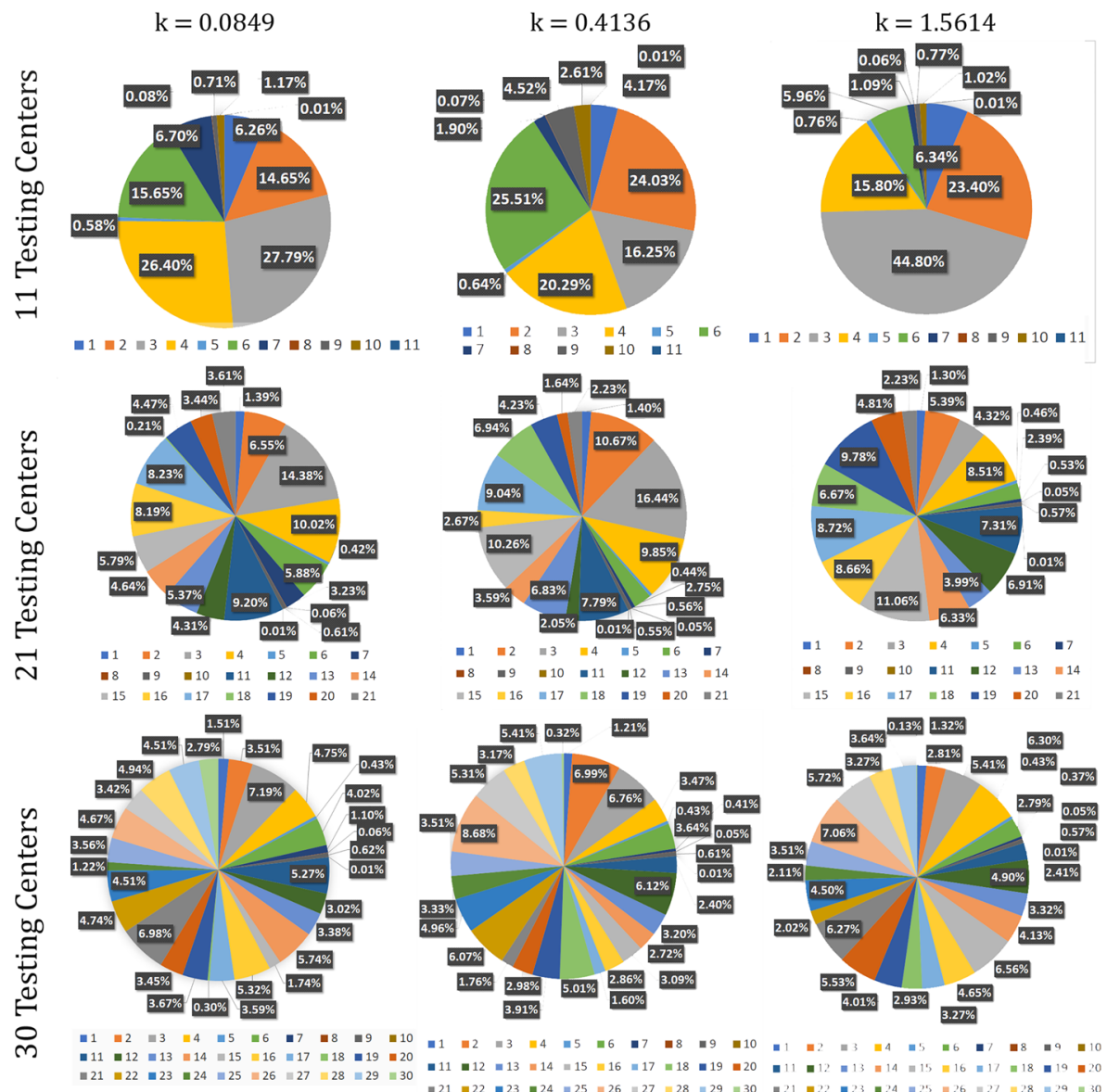

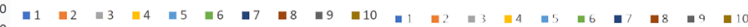
- $21=22=23=24=25=26=27=28=29=30$

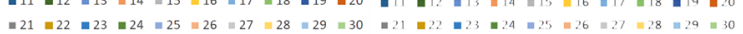

Fig. 2 Proportion of test kits for various number of testing centers against different values of $k$. These testing centers are located in (1) Research Institute for Tropical Medicine (RITM); (2) San Lazaro Hospital; (3) University of the Philippines - National Institutes of Health; (4) Lung Center of the Philippines; (5) Baguio General Hospital and Medical Center; (6) The Medical City, Pasig City; (7) St. Luke's Medical Center, Quezon City; (8) Bicol Public Health Laboratory; (9) Western Visayas Medical Center; (10) Vicente Sotto Memorial Medical Center; (11) Southern Philippines Medical Center; (12) Caloocan City; (13) Cavite; (14) Mandaluyong City; (15) Makati City; (16) Laguna; (17) Taguig City; (18) Pasay City; (19) Rizal; (20) Bulacan; (21) Las Piñas City; (22) Parañaque City; (23) Marikina City; (24) Malabon City; (25) Valenzuela City; (26) Navotas City; (27) Pangasinan; (28) Batangas; (29) Negros Occidental; (30) San Juan City

center $j$ as shown in Fig. 1. In the country, there have been 38640 tests conducted as of April 11,2020. We considered the optimal allocation for $k=1.5614$ and compare it to the actual distribution of positive cases tested in these various testing centers as shown in Table 2. Since the various testing centers have different dates of accreditation as depicted in the table, we can see a huge discrepancy to the allocation proposed in this study. 


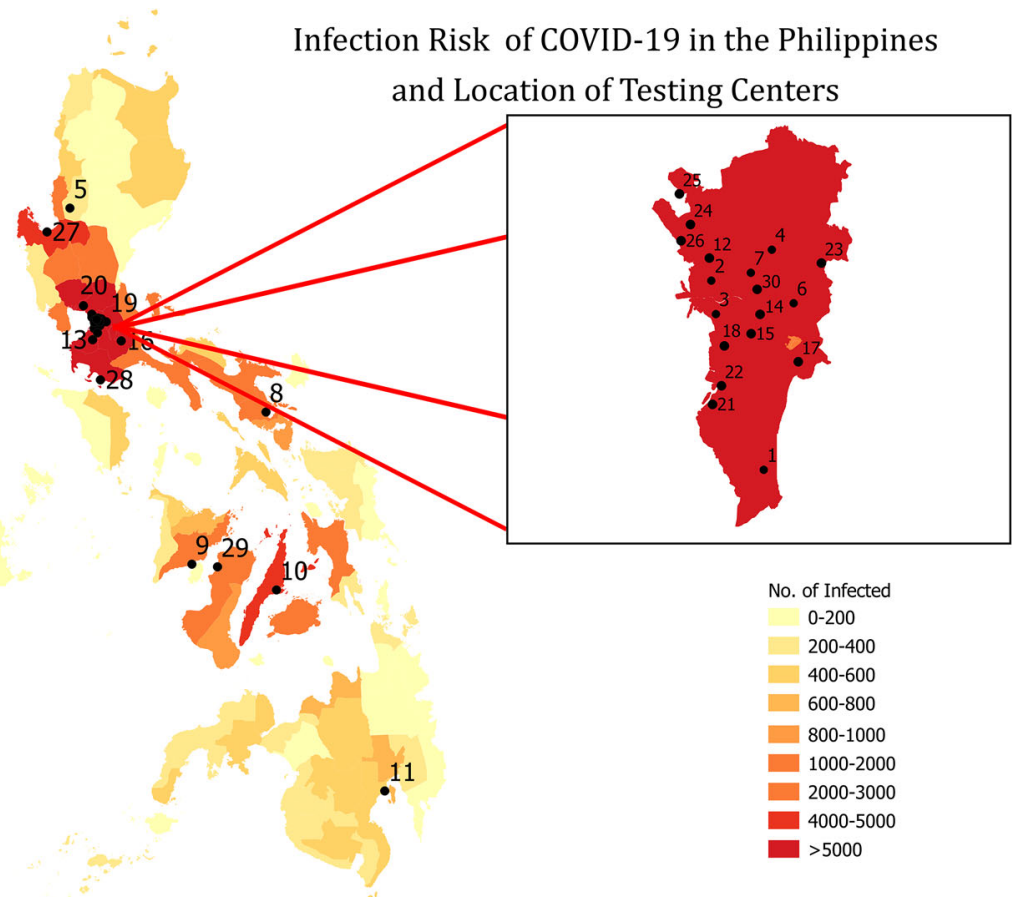

Fig. 3 Locations of the 11 testing centers accredited by the World Health Organization as of 12 April 2020 and the 19 additional testing centers. The following are the testing centers and are shown in the map: (1) Research Institute for Tropical Medicine (RITM); (2) San Lazaro Hospital; (3) University of the Philippines - National Institutes of Health (UP-NIH); (4) Lung Center of the Philippines; (5) Baguio General Hospital and Medical Center (BGHMC); (6) The Medical City, Pasig City (TMC); (7) St. Luke's Medical Center, Quezon City; (8) Bicol Public Health Laboratory (BPHL); (9) Western Visayas Medical Center (WVMC); (10) Vicente Sotto Memorial Medical Center (VSMMC); (11) Southern Philippines Medical Center (SPMC); (12) Caloocan City; (13) Cavite; (14) Mandaluyong City; (15) Makati City; (16) Laguna; (17) Taguig City; (18) Pasay City; (19) Rizal; (20) Bulacan; (21) Las Piñas City; (22) Parañaque City; (23) Marikina City; (24) Malabon City; (25) Valenzuela City; (26) Navotas City; (27) Pangasinan; (28) Batangas; (29) Negros Occidental; (30) San Juan City

Assuming that $100 \%$ of all test kits have been utilized, we noticed that the number of test kits allocated to testing centers in Metro Manila (which has the highest number of cases in both actual and projected number of infected individuals) is greater than that of the actual data. At the same time, the distribution outside the region is fairly proportional to the estimated number of individuals on each testing center's location. For instance, RITM gained an $89.48 \%$ positive tests among all the positive cases as of April 11. This is due to the fact that RITM was the first testing center accredited by DOH. If the proposed model will be used, about $6.34 \%$ of test kits should be allotted to RITM. Following the trend of positivity from the actual data, medical workers 
Table 2 Actual positivity rate of individuals tested in the Philippines as of April 11 [19], computed allocation of test kits from our NLP model with $k=1.5614$, and date of accreditation of the testing center

\begin{tabular}{llll}
\hline & Actual (as of April 11) & Computed $(k=1.5614)$ & Date accredited \\
\hline RITM & $89.48 \%$ & $6.34 \%$ & $3 / 17 / 2020$ \\
San Lazaro & $1.16 \%$ & $23.40 \%$ & $3 / 27 / 2020$ \\
UP-NIH & $2.42 \%$ & $44.80 \%$ & $3 / 27 / 2020$ \\
Lung Center & $1.38 \%$ & $15.18 \%$ & $3 / 27 / 2020$ \\
BGHMC & $0.71 \%$ & $0.76 \%$ & $3 / 27 / 2020$ \\
TMC & $0.00 \%$ & $5.96 \%$ & $4 / 6 / 2020$ \\
St. Luke's & $1.38 \%$ & $1.09 \% 6$ & $4 / 5 / 2020$ \\
BPHL & $0.08 \%$ & $0.06 \%$ & $4 / 5 / 2020$ \\
WVMC & $0.73 \%$ & $0.77 \%$ & $3 / 31 / 2020$ \\
VSMMC & $0.57 \%$ & $1.02 \%$ & $3 / 27 / 2020$ \\
SPMC & $2.09 \%$ & $0.01 \%$ & $3 / 27 / 2020$ \\
\hline
\end{tabular}

from RITM have a tendency to be overworked. Whereas in the proposed model, the distribution is contingent on the density of the area, and the test kits to be distributed will be proportional to the positive cases in the municipality.

With these test kits being allocated in an optimal and equitable way, more communities in the country can have an easy access to testing, which leads to an early detection of infection and can speed up the isolation of infected individuals. This will result in lowering the spread of the disease and "flattening" the epidemiological curve.

In terms of testing capacity in the country, we note that if only 11 testing centers are to be used for testing COVID-19 patients, several testing centers will be overworked and there would be longer turn-around-time in releasing test results, especially if mass testing will push through. For instance, let us assume that 300,000 test kits will be distributed. Based from the results of our model, UP-NIH will receive an average of almost 89,000 test kits, across the different values of dispersal length. This implies that UP-NIH needs more than 1000 days to conduct the whole testing process. This translates to more than 2 years. We recommend that additional testing centers should be placed in strategic locations, that is, in the communities with high density of infected individuals as shown in Fig. 3. If we consider the proposed 30 testing centers, UP-NIH will receive almost 19,000 test kits, and this translates to 245 days to complete the tests (Appendix Tables Table 3 and 4). Hence, increasing the number of testing centers from 11 to 30 will result in a drastic decrease in the number of days needed to complete all the tests. This means that as more testing centers operate, more tests can be conducted per day. This will lead to faster completion of all the tests. 


\section{Conclusion}

Our results suggested an optimal allocation of COVID-19 test kits among the DOHaccredited testing centers in the Philippines. The proposed percentage allocations per testing center gave an equal opportunity for all estimated infected people all over the country to have an initial testing for the virus infection. With our allocations, we utilized $100 \%$ of the limited test kits available to the country, and distributed them optimally to all testing centers available in the country. However, it is recommended that additional testing centers should be placed in strategic locations to reach more infected individuals and hasten the testing process in the country.

Using our model on test kit allocation, especially in resource-constrained countries, can be helpful to policymakers in making a decision on how to distribute available COVID-19 test kits among accredited testing centers while utilizing the limited amount of test kits. Authorities may apply this model not just to countries, but to their respective communities as well, if the public health system and situation follow the same assumptions and goal of the model. Having an optimal distribution of test kits will help a lot of agencies, e.g., healthcare system, local government units, and other institutions helping the policymakers fight this pandemic. By optimally allocating the test kits, no resources are wasted and all are utilized properly and equitably.

In addition, policymakers may also assess the feasibility of the percentage allocation depending on their current situation (e.g., epidemiological and socio-economic). The model exhausts all available test kits but the complexity of test kit resource dynamics determines the limitation of our study. The current model limits test kits to only one per individual (initial testing), and only considers an estimated number of infected individuals (symptomatic patients) due to the assumed limited amount of resources. The proposed optimal allocation from our model also holds an assumption that it will be distributed and utilized all at the same time. Future studies may consider various sets of population depending on the available amount of test kits to their country. It should also incorporate individuals needing multiple tests, effectiveness of test kits, and transport time for test kit delivery. In conclusion, our model offers an optimal way to allocate the COVID-19 test kits among testing centers in the country, with every (estimated) infected individual having an equal opportunity of getting tested. However, a larger number of both test kits and testing centers are still more ideal to increase the number of individuals tested to aid in "flattening" the epidemic curve.

\section{Supplementary Files}

The Microsoft Excel file containing the municipality/city data, GPS location, and population density-based number of infections, and $\mathrm{R}$ files used for the model simulation can be found online at github.com/alvinizer/optimalallocationPH/.

Funding JFR is supported by the Abdus Salam International Centre for Theoretical Physics Associateship Scheme. 


\section{Appendix 1. Optimal solution for 21 testing centers}

Table 3 Proportion of test kits to be distributed in 21 testing centers

\begin{tabular}{|c|c|c|c|c|c|}
\hline Testing center & $k=1.5614$ & $k=0.4136$ & $k=0.0849$ & Range & Daily limit \\
\hline RITM & $1.3 \%$ & $1.4 \%$ & $1.39 \%$ & {$[1.30 \%, 1.40 \%]$} & 900 \\
\hline San Lazaro & $5.39 \%$ & $10.67 \%$ & $6.55 \%$ & {$[5.39 \%, 10.67 \%]$} & 100 \\
\hline UP-NIH & $4.32 \%$ & $16.44 \%$ & $14.38 \%$ & {$[4.32 \%, 16.44 \%]$} & 80 \\
\hline Lung Center & $8.51 \%$ & $9.85 \%$ & $10.02 \%$ & {$[8.51 \%, 10.02 \%]$} & 100 \\
\hline BGHMC & $0.46 \%$ & $0.44 \%$ & $0.42 \%$ & {$[0.42 \%, 0.46 \%]$} & 150 \\
\hline TMC & $2.39 \%$ & $2.75 \%$ & $5.88 \%$ & {$[2.39 \%, 5.88 \%]$} & $100 *$ \\
\hline St. Luke's, QC & $0.53 \%$ & $0.56 \%$ & $3.23 \%$ & {$[0.53 \%, 3.23 \%]$} & $100^{*}$ \\
\hline BPHL & $0.05 \%$ & $0.05 \%$ & $0.06 \%$ & {$[0.05 \%, 0.06 \%]$} & $100 *$ \\
\hline WVMC & $0.57 \%$ & $0.55 \%$ & $0.61 \%$ & {$[0.55 \%, 0.61 \%]$} & 80 \\
\hline VSMMC & $0.01 \%$ & $0.01 \%$ & $0.01 \%$ & {$[0.01 \%, 0.01 \%]$} & 240 \\
\hline SPMC & $7.31 \%$ & $7.79 \%$ & $9.2 \%$ & {$[7.31 \%, 9.20 \%]$} & 100 \\
\hline Caloocan City & $6.91 \%$ & $2.05 \%$ & $4.31 \%$ & {$[2.05 \%, 6.91 \%]$} & $100 *$ \\
\hline Cavite & $3.99 \%$ & $6.83 \%$ & $5.37 \%$ & {$[3.99 \%, 6.83 \%]$} & $100 *$ \\
\hline Mandaluyong City & $6.33 \%$ & $3.59 \%$ & $4.64 \%$ & {$[3.59 \%, 6.33 \%]$} & $100 *$ \\
\hline Makati City & $11.06 \%$ & $10.26 \%$ & $5.79 \%$ & {$[5.79 \%, 11.06 \%]$} & $100 *$ \\
\hline Laguna & $8.66 \%$ & $2.67 \%$ & $8.19 \%$ & {$[2.67 \%, 8.66 \%]$} & $100 *$ \\
\hline Taguig City & $8.72 \%$ & $9.04 \%$ & $8.23 \%$ & {$[8.23 \%, 9.04 \%]$} & $100 *$ \\
\hline Pasay City & $6.67 \%$ & $6.94 \%$ & $0.21 \%$ & {$[0.21 \%, 6.94 \%]$} & $100 *$ \\
\hline Rizal & $9.78 \%$ & $4.23 \%$ & $4.47 \%$ & {$[4.23 \%, 9.78 \%]$} & $100^{*}$ \\
\hline Bulacan & $4.81 \%$ & $1.64 \%$ & $3.44 \%$ & {$[1.64 \%, 4.81 \%]$} & $100 *$ \\
\hline Las Piñas City & $2.23 \%$ & $2.23 \%$ & $3.61 \%$ & {$[2.23 \%, 3.61 \%]$} & $100^{*}$ \\
\hline
\end{tabular}

* - Assumed maximum tested individuals per day

\section{Appendix 2. Optimal solution for $\mathbf{3 0}$ testing centers}

Table 4 Proportion of test kits to be distributed in 30 testing centers

\begin{tabular}{llllll}
\hline Testing center & $k=1.5614$ & $k=0.4136$ & $k=0.0849$ & Range & Daily limit \\
\hline $\begin{array}{l}\text { Research institute for trop- } \\
\text { ical medicine (RITM) }\end{array}$ & $1.32 \%$ & $1.21 \%$ & $1.51 \%$ & {$[1.21 \%, 1.51 \%]$} & 900 \\
San Lazaro Hospital & $2.81 \%$ & $6.99 \%$ & $3.51 \%$ & {$[2.81 \%, 6.99 \%]$} & 100 \\
UP-NIH & $5.41 \%$ & $6.76 \%$ & $7.19 \%$ & {$[5.41 \%, 7.19 \%]$} & 80 \\
Lung Center of the Philippines & $6.3 \%$ & $3.47 \%$ & $4.75 \%$ & {$[3.47 \%, 6.30 \%]$} & 100 \\
BGHMC & $0.43 \%$ & $0.43 \%$ & $0.43 \%$ & {$[0.43 \%, 0.43 \%]$} & 150 \\
TMC & $2.79 \%$ & $3.64 \%$ & $4.02 \%$ & {$[2.79 \%, 4.02 \%]$} & $100 *$
\end{tabular}


Table 4 (continued)

\begin{tabular}{|c|c|c|c|c|c|}
\hline Testing center & $k=1.5614$ & $k=0.4136$ & $k=0.0849$ & Range & Daily limit \\
\hline $\begin{array}{l}\text { St. Luke's Medical Center, } \\
\text { Quezon City }\end{array}$ & $0.37 \%$ & $0.41 \%$ & $1.1 \%$ & {$[0.37 \%, 1.10 \%]$} & $100 *$ \\
\hline BPHL & $0.05 \%$ & $0.05 \%$ & $0.06 \%$ & {$[0.05 \%, 0.06 \%]$} & $100 *$ \\
\hline WVMC & $0.57 \%$ & $0.61 \%$ & $0.62 \%$ & {$[0.57 \%, 0.62 \%]$} & 80 \\
\hline VSMMC & $0.01 \%$ & $0.01 \%$ & $0.01 \%$ & {$[0.01 \%, 0.01 \%]$} & 240 \\
\hline SPMC & $2.41 \%$ & $2.4 \%$ & $5.27 \%$ & {$[2.40 \%, 5.27 \%]$} & 100 \\
\hline Caloocan City & $4.9 \%$ & $6.12 \%$ & $3.02 \%$ & {$[3.02 \%, 6.12 \%]$} & $100 *$ \\
\hline Cavite & $3.32 \%$ & $3.2 \%$ & $3.38 \%$ & {$[3.20 \%, 3.38 \%]$} & $100 *$ \\
\hline Mandaluyong City & $4.13 \%$ & $2.72 \%$ & $5.74 \%$ & {$[2.72 \%, 5.74 \%]$} & $100 *$ \\
\hline Makati City & $6.56 \%$ & $3.09 \%$ & $1.74 \%$ & {$[1.74 \%, 6.56 \%]$} & $100 *$ \\
\hline Laguna & $4.65 \%$ & $2.86 \%$ & $5.32 \%$ & {$[2.86 \%, 5.32 \%]$} & $100^{*}$ \\
\hline Taguig City & $3.27 \%$ & $1.6 \%$ & $3.59 \%$ & {$[1.60 \%, 3.59 \%]$} & $100 *$ \\
\hline Pasay City & $2.93 \%$ & $5.01 \%$ & $0.3 \%$ & {$[0.30 \%, 5.01 \%]$} & $100 *$ \\
\hline Rizal & $4.01 \%$ & $3.91 \%$ & $3.67 \%$ & {$[3.67 \%, 4.01 \%]$} & $100 *$ \\
\hline Bulacan & $5.53 \%$ & $2.98 \%$ & $3.45 \%$ & {$[2.98 \%, 5.53 \%]$} & $100 *$ \\
\hline Las Piñas City & $6.27 \%$ & $1.76 \%$ & $6.98 \%$ & {$[1.76 \%, 6.98 \%]$} & $100 *$ \\
\hline Parañaque City & $2.02 \%$ & $6.07 \%$ & $4.74 \%$ & {$[2.02 \%, 6.07 \%]$} & $100 *$ \\
\hline Marikina City & $4.50 \%$ & $4.96 \%$ & $4.51 \%$ & {$[4.50 \%, 4.96 \%]$} & $100 *$ \\
\hline Malabon City & $2.11 \%$ & $3.33 \%$ & $1.22 \%$ & {$[1.22 \%, 3.33 \%]$} & $100 *$ \\
\hline Valenzuela City & $3.51 \%$ & $3.51 \%$ & $3.56 \%$ & {$[3.51 \%, 3.56 \%]$} & $100 *$ \\
\hline Navotas City & $7.06 \%$ & $8.68 \%$ & $4.67 \%$ & {$[4.67 \%, 8.68 \%]$} & $100 *$ \\
\hline Pangasinan & $5.72 \%$ & $5.31 \%$ & $3.42 \%$ & {$[3.42 \%, 5.72 \%]$} & $100 *$ \\
\hline Batangas & $3.27 \%$ & $3.17 \%$ & $4.94 \%$ & {$[3.17 \%, 4.94 \%]$} & $100^{*}$ \\
\hline Negros Occidental & $3.64 \%$ & $5.41 \%$ & $4.51 \%$ & {$[3.64 \%, 5.41 \%]$} & $100 *$ \\
\hline San Juan City & $0.13 \%$ & $0.32 \%$ & $2.79 \%$ & {$[0.13 \%, 2.79 \%]$} & $100^{*}$ \\
\hline
\end{tabular}

* - Assumed maximum tested individuals per day

\section{References}

1. Philippine Statistics Authority. Philippine population density (Based on the 2015 Census of Population). 2020. https://psa.gov.ph/content/philippine-population-density-based-2015-census-population (visited on 04/11/2020)

2. Bradley SP, Hax AC, Magnanti TL (1977) Applied mathematical programming. Addison-Wesley Publishing Company. isbn: 9780201004649 . https://books.google.com.ph/books?id=MSWdWv3Gn5cC

3. Buhat CAH et al (2017) Stochasticity in the parasite-driven trait evolution of competing species masks the distinctive consequences of distance metrics. Processes 5(4):74

4. Calonzo A Coronavirus disease 2019 (COVID-19) Situation report-46. 2020. https://www. who.int/docs/default-source/coronaviruse/situationreports/20200306-sitrep-46-covid-19.pdf? sfvrsn=96b04adf_4 (visited on 04/11/2020)

5. Choi J (2020) Covid-19 in South Korea. In: Postgraduate medical journal 96. https://doi.org/10.1136/ postgradmedj-2020-137738

6. Delamater P et al (2019) Complexity of the basic reproduction number (R0). Emerging Infectious Diseases 25:1-4. https://doi.org/10.3201/eid2501.171901 
7. Elhadi M et al (2020) Concerns for low-resource countries, with under-prepared intensive care units, facing the COVID-19 pandemic. In: Infection, disease health. https://doi.org/10.1016/j.idh. 2020.05.008

8. Elliot JM (1971) Some methods for the statistical analysis of samples of benthic invertebrate. In: Freshwater biological association scientific

9. Ferguson $\mathrm{N}$ et al (2020) Report 9: Impact of non-pharmaceutical interventions (NPIs) to reduce COVID-19 mortality and healthcare demand. In:. https://doi.org/10.25561/77482

10. Ha KM (2020) A lesson learned from the outbreak of COVID-19 in korea. In: Indian journal of microbiology. https://doi.org/10.1007/s12088-020-00882-7

11. Hamidi S, Sabouri S, Ewing R (2020) Does density aggravate the COVID-19 pandemic?: early findings and lessons for planners. In: Journal of the American Planning Association, pp 1-15. https://doi.org/10.1080/01944363.2020.1777891

12. Department of Health. 2020. https://www.doh.gov.ph/2019-nCov/FAQs (visited on 07/25/2020)

13. Department of Health. 2020. https://www.doh.gov.ph/doh-press-release/\%20COVID-DAILYTESTING-CAPACITY-INCREASES-NUMBERS-TO-IMPROVE-FURTHER-TO-10K (visited on 07/25/2020)

14. Kissler S et al (2020) Projecting the transmission dynamics of SARS-cov-2 through the postpandemic period. In: Science 368. https://doi.org/10.1126/science.abb5793

15. Lee D, Heo K, Seo Y (2020) COVID-19 In South korea: lessons for developing countries. In: World development. https://doi.org/10.1016/j.worlddev.2020.105057

16. Li R et al (2020) Substantial undocumented infection facilitates the rapid dissemination of novel coronavirus (SARS-cov2). In: Science 368. https://doi.org/10.1126/science.abb3221

17. Monnat S, Cheng KJ (2020) Geographic disparities in COVID-19 testing: an urgent call to action in

18. Nichat M (2013) Landmark based shortest path detection by using Dijkestra Algorithm and Haversine Formula. In: International journal of engineering research and applications(IJERA) ISSN: 2248-9622 vol 3, Issue 3, may-jun 2013, pp 162

19. World Health Organization. 2020. https://www.who.int/docs/default-source/wpro---documents/ countries/philippines/emergencies/covid-19/who-phl-sitrep-18-covid-19-13apr2020.pdf? sfvrsn=f4091764_2 (visited on 07/25/2020)

20. World Health Organization. WHO Director-General's opening remarks at the media briefing on COVID-19 - 11 March 2020. 2020. https://www.who.int/dg/speeches/detail/who-director-general-sopening-remarks-at-the-media-briefing-on-covid-19-11-march-2020 (visited on 04/12/2020)

21. Prather K, Wang C, Schooley R (2020) Reducing transmission of SARS-cov-2. In: Science 368. https://doi.org/10.1126/science.abc6197

22. Quintos P (2020) The Philippines' COVID-19 response: symptoms of deeper malaise in the Philippine health system in

23. Riou J, Althaus CL (2020) Pattern of early human-to-human transmission of Wuhan 2018 novel coronavirus (2019-nCoV December 2019 to January 2020). In: Euro surveillance 25(4)

24. Sanche $S$ et al (2020) High contagiousness and rapid spread of severe acute respiratory syndrome coronavirus 2. In: Emerg infect dis 2

25. Santiago I (2020) Trends and innovations in biosensors for COVID-19 mass testing. In: Chembiochem. https://doi.org/10.1002/cbic.202000250

26. Schlickeiser R, Schlickeiser F (2020) A Gaussian model for the time development of the Sars-Cov2 corona pandemic disease. Predictions for Germany made on 30 March 2020. Physics 2:164-170. https://doi.org/10.3390/physics2020010

27. Studdert D, Hall M (2020) Disease control civil liberties, and mass testing - calibrating restrictions during the covid-19 pandemic. In: The New England journal of medicine 383. https://doi.org/10.1056/ NEJMp2007637

28. Thwe, Win S (2018) Cities guide system using spherical law. In: International journal of scientific and research publications (IJSRP) 8. https://doi.org/10.29322/IJSRP.8.11.2018.p8355

29. Vanden Broecke B et al (Aug. 2019) Relationship between population density and viral infection: a role for personality?. In: Ecology and evolution. https://doi.org/10.1002/ece3.5541

30. Ward S et al (2020) Clinical testing for covid-19. In: Journal of allergy and clinical immunology 146. https://doi.org/10.1016/j.jaci.2020.05.012

31. Wilson DP, Blower SM (2005) Designing equitable antiretroviral allocation strategies in resourceconstrained countries. In: PLOS Medicine 2 
32. Zhu N et al (2020) A novel coronavirus from patients with pneumonia in China, 2019. In: New england journal of medicine 382. https://doi.org/10.1056/NEJMoa2001017

Publisher's Note Springer Nature remains neutral with regard to jurisdictional claims in published maps and institutional affiliations. 